\title{
Emilio Huerta*
}

Ester Martinez-Ros**

Vicente Salas Fumás ${ }^{* * *}$

\section{UN LUGAR PARA LA EMPRESA EN LA POLÍTICA INDUSTRIAL}

Este artículo establece los déficits que se observan en la inversión en recursos intangibles (capital directivo y capital organizacional), en las empresas españolas cuando se comparan con las empresas de los países más avanzados de la Unión Europea. Se sugiere que «la politica industrial» se oriente a actuar sobre estos problemas del ámbito empresarial y se centre más en las cuestiones de «cómo» deben innovar y competir las empresas que «dónde» deben competir, para diseñar las palancas que estimulen los cambios.

\section{A venue for the companies in the industrial policy}

This paper establishes the deficits that are observed in the investment in intangible resources (managerial capital and organizational capital) in Spanish companies when compared with companies in the most advanced countries of the European Union. The paper suggests that the Industrial Policy should be oriented to deal with these problems in the business environment and to focus more on the questions of how companies should compete rather than where they should compete.

Palabras clave: inversión en intangibles, capital directivo y capital organizacional, productividad, politicas públicas.

Keywords: investment in intangibles, managerial capital and organizational capital, productivity, public policies.

JEL: E22, J24, J53, K21.

\footnotetext{
* Universidad Pública de Navarra.

Contacto: ehuerta@unavarra.es

Agradece el apoyo financiero del Ministerio de Economía, Industria

y Competitividad a través del proyecto ECO2017-86305-C4-4-R

(AEI/FEDER, UE).

** Universidad Carlos III de Madrid.

Contacto: ester.martinez@uc3m.es

*** Universidad de Zaragoza.

Contacto: vsalas@unizar.es

Agradece el apoyo financiero de CREVALOR (S42_17R, Gobierno de

Aragón -España-y FEDER 2014-2020 «Construyendo Europa

desde Aragón») y del proyecto ECO2017-86305-C4-3-R: «Contribuciones

al Diseño de Modelos de Negocio Socialmente Sostenibles» Ministerio de

Economía, Industria y Competitividad -España-y FEDER.

Versión de febrero de 2021.

https://doi.org/10.32796/ice.2021.919.7173
} 


\section{Introducción}

La crisis sanitaria y económica que ha desencadenado la COVID-19, ha creado unas condiciones propicias para que los Estados nacionales pongan en marcha políticas públicas dirigidas a aumentar la autosuficiencia de bienes y servicios básicos, reducir la vulnerabilidad de sus economías a los shocks externos y fortalecer su resiliencia. Pero en el escenario español, la crisis actual no ha hecho sino profundizar las debilidades de nuestro tejido productivo. Un rumbo mediocre de crecimiento de la productividad de las empresas y de la economía son síntomas profundos de la debilidad que muestra la trayectoria de la economía española desde hace tiempo. Parece que faltan políticas públicas, programas de intervención, dirección y compromisos entre los protagonistas públicos y privados para avanzar hacia una economía más próspera y con mayor capacidad para adaptarse a un mundo crecientemente complejo.

En este contexto, la Unión Europea (UE) ha puesto en marcha un programa de estímulos económicos sin precedentes, conocido como programa Next Generation EU (NGEU) con el que se pretende acelerar la transformación de las economías europeas, reduciendo las emisiones de carbono y avanzando en la digitalización, para conseguir que sean más productivas, más sostenibles y más inclusivas.

Por ello, reflexionar sobre cómo intervenir para corregir la trayectoria y los equilibrios de las empresas y la economía no solo resulta urgente para aprovechar bien el estímulo de demanda de los fondos europeos sino también, resulta clave para actuar sobre la oferta si lo que nos preocupa es la prosperidad de nuestro país en el medio y largo plazo.

¿Cómo debería intervenir el Gobierno, qué programas y políticas desarrollar para reducir la intensidad de los procesos y obstáculos que lastran el crecimiento de la economía española? ¿Cómo se refuerza a las empresas, se potencian los sectores y tecnologías más atractivas y se ubican las empresas españolas en las etapas de las cadenas globales de valor más interesantes y de mayor potencial? La cuestión ahora no es tanto el porqué intervenir, hay voluntad de actuar y fondos disponibles, sino cómo intervenir. ¿Qué tipo de política industrial hay que desarrollar?

La política industrial, en su significado más amplio de políticas públicas de corte microeconómico dirigidas a influir en la distribución relativa de la actividad entre sectores económicos a favor de aquellos más dinámicos y productivos, deberá encontrar su protagonismo en este nuevo entorno. Estos nuevos desafíos requerirán, previsiblemente, ampliar el espectro de políticas públicas de carácter transversal en I+D+i, formación y modernización productiva, para incorporar aspectos de calidad medioambiental, adopción y uso eficiente de innovaciones en el ámbito de la digitalización, así como consideraciones de equidad distributiva.

Acompañando a los objetivos y prioridades estratégicos para el conjunto de la UE, el programa NGEU plantea la necesidad de valorar los mecanismos a través de los cuales se va a gestionar e instrumentar el cambio transformador al que se aspira. En este contexto surge de nuevo el debate alrededor de los papeles respectivos del Estado, el mercado y las empresas en la conducción del proceso. Parece que esta vez se apuesta por parte de empresas y Administraciones públicas por lo que se ha denominado colaboración pública-privada. El interés ahora está en los entes ejecutores de los planes y los programas, de manera que tan importante para el éxito final de las grandes transformaciones previstas será el qué se va a hacer, como el cómo se va a hacer.

Nuestro trabajo se centra en el análisis de los problemas de competitividad que presenta la economía e industria española, centrando el estudio en el análisis de sus empresas. Queremos centrar el foco en las limitaciones y barreras que tienen las empresas españolas para crear más valor y riqueza, para ser más competitivas. Este enfoque resulta novedoso porque la mayor parte de los estudios académicos (Andrés \& Doménech, 2015) se centran en el estudio del entorno institucional, la regulación y el funcionamiento de los mercados y los sectores cuando se analizan las debilidades de la economía 
española. Pensamos que los problemas de falta de innovación, limitada internacionalización y baja productividad comienzan en las empresas.

Dentro de este marco general de reflexión, el objetivo de este trabajo es triple. En primer lugar, aportar información sobre el sector empresarial español que debe de servir para valorar la situación de partida a la que deberán adaptarse los proyectos transformadores impulsados por la UE, incluidas las políticas industriales (microeconómicas) para asegurar el mejor resultado final posible. En segundo lugar, utilizar el ejemplo de las políticas de apoyo a la innovación tecnológica de la UE como referencia de los nuevos programas de actuación pública a los que tendrán que adaptarse las empresas españolas. Y, por último, ofrecer unas recomendaciones de intervención pública vinculadas con las necesidades que tienen las empresas españolas.

El principal propósito de nuestro trabajo es el de abrir e iluminar el interior de la caja negra de las empresas que la política industrial ha mantenido relativamente cerrada. Esto significa haber dejado fuera del interés estricto de la política industrial aspectos que investigaciones recientes han demostrado ser muy relevantes para entender la eficiencia y el dinamismo empresarial, como son: la calidad de la gestión, la organización del trabajo, las políticas de recursos humanos o la voz y participación de los trabajadores (Bloom \& Van Reenen, 2007, 2010; Bloom et al., 2014; Giorcelli, 2019).

El resto del trabajo se organiza como sigue. En el apartado segundo se presentan algunos datos agregados sobre la evolución, dinamismo, del sector empresarial español, sociedades no financieras, en los últimos veinte años en los que quedan patentes signos de bajo crecimiento de la productividad, alta vulnerabilidad a los shocks externos y baja capacidad adaptativa a través de la renovación del tejido productivo. En el apartado tercero se presentan aspectos cualitativos del tejido empresarial español, relacionados con el capital organizacional y directivo de las empresas que pueden explicar los resultados del agregado. El cuarto se centra en la relación Estado-mercado en el ámbito del fomento de la innovación tecnológica que considera a la vez aspectos de eficiencia y de equidad en el reparto de la renta creada. El apartado quinto resume algunas de las recomendaciones sobre políticas públicas que emergen de los diagnósticos anteriores. Finalmente, las conclusiones sintetizan las ideas principales de toda la exposición.

\section{El sector empresarial español}

\section{Dinamismo y productividad}

En este apartado se presentan algunas evidencias sobre el comportamiento del sector empresarial español en los últimos veinte años, cuando España forma parte de la zona euro. En línea con los objetivos más representativos de la política industrial, interesa conocer el dinamismo y la productividad de las empresas españolas durante las dos décadas en las que, la pertenencia a la zona euro, debería asegurarles unas condiciones financieras similares a las del resto de economías que comparten la moneda común. Los indicadores de dinamismo empresarial utilizados en la exposición son indicadores de output (VAB, valor añadido bruto) e inputs (trabajo y capital) para el conjunto de las sociedades no financieras (SNF) en España, y las tasas de natalidad y mortalidad de empresas. La productividad se mide en términos de productividad total de los factores (PTF). Las SNF incluyen al conjunto de empresas personas jurídicas societarias, en todos los sectores de la economía excepto el financiero; quedan fuera del sector empresarial las empresas no financieras sin personalidad jurídica societaria, principalmente autónomos sin asalariados, cuya actividad se contabiliza en el sector institucional de los hogares.

\section{Producción y productividad}

La Figura 1 muestra la evolución del índice, base 1 en el año 2000 , de volumen de producción y cantidades de recursos de trabajo y capital empleados en la misma para el conjunto de SNF en España, entre los años 2000 y 2019 (año previo a la pandemia de la COVID-19). La 


\section{FIGURA 1}

ÍNDICES DE EVOLUCIÓN DEL OUTPUT (VAB) E INPUTS (TRABAJO Y CAPITAL) A EUROS CONSTANTES DEL AÑO 2000 PARA EL CONJUNTO DE SNF EN ESPAÑA

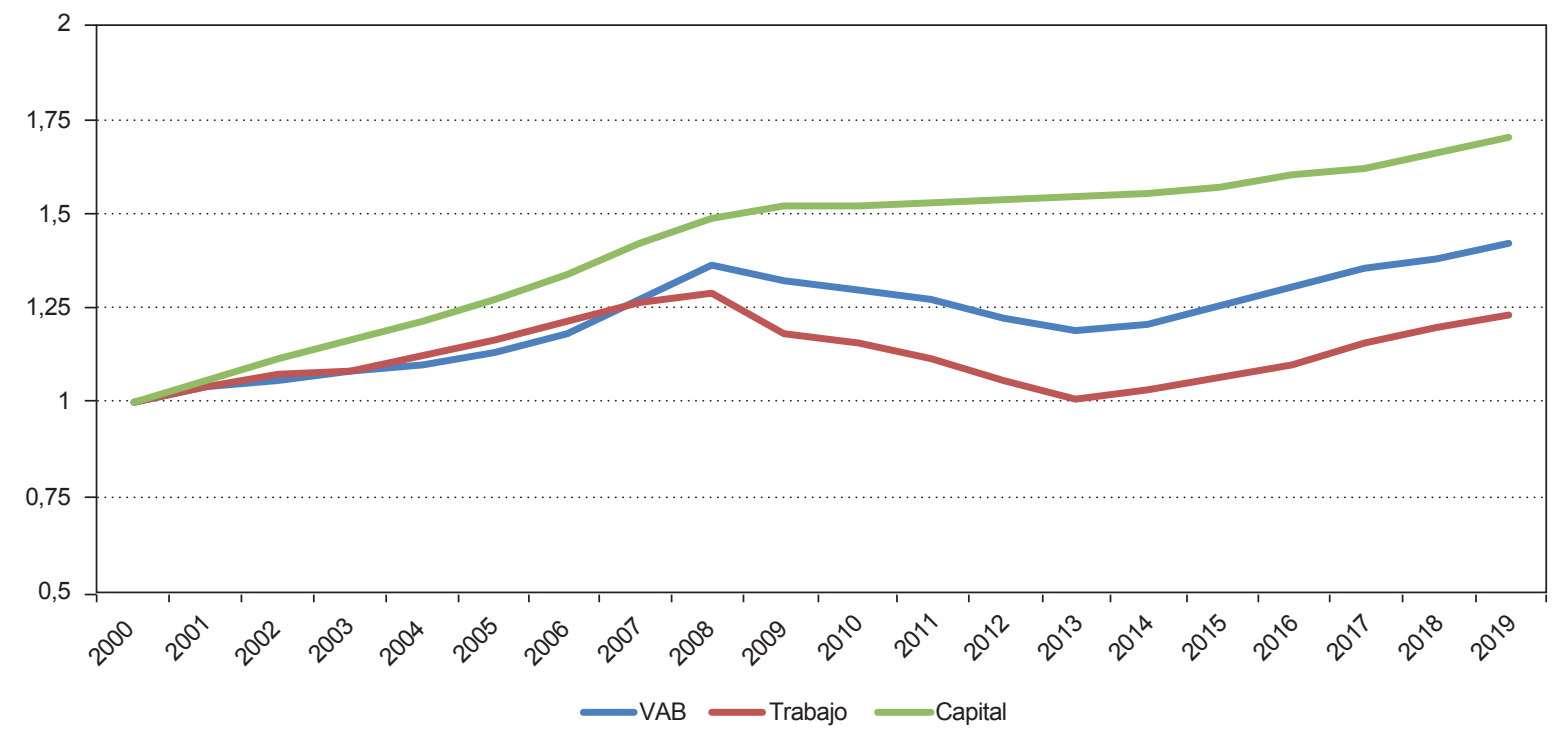

FUENTE: Elaboración propia a partir de la contabilidad nacional (sector institucional de las SNF) y de los índices de precios y salarios calculados con datos del INE.

producción se mide por el VAB a precios constantes del año 2000 (valores corrientes divididos por el índice del deflactor del PIB con base 1 en el año 2000). El índice de cantidad de trabajo se calcula a partir de los gastos de personal anuales de las SNF publicados por la contabilidad nacional, divididos por un índice de evolución de los salarios a partir del coste laboral medio por trabajador en España, calculado por el índice (base 1 en el año 2000). El índice de cantidad de capital se calcula en dos etapas; primero se estima el stock de capital productivo a precios corrientes de reposición a partir del método de inventario permanente y con flujos anuales de inversión en capital y dotación de amortizaciones de las SNF; posteriormente, se estima el stock de capital a precios constantes del año 2000 utilizando el deflactor de la formación de capital.

En el año 2000, las SNF generan en España VAB por valor de 326.025 millones de euros, pagan salarios por importe de 206.805 millones de euros (63\% del VAB) y disponen de un stock de capital para producir en España de 637.767 millones de euros. La crisis financiera de 2009 interrumpe una fase de crecimiento sostenido del output de las SNF en España que incluso se acelera entre 2005 y 2008, de manera que en 2008 el output es un $36 \%$ mayor que en el año 2000. La crisis cambia la tendencia y en el periodo 2009-2013 el output es hasta un $12 \%$ menor que en el pico de 2008. A partir de 2014, empieza una senda de recuperación de manera que tres años más tarde, en 2017, se recuperan los niveles de 2008 y, en 2019, el output es un $42 \%$ superior al del año 2000 (equivalente a algo menos del $2 \%$ de crecimiento constante acumulativo anual del 2 \%). En 2020, el output vuelve a contraerse esta vez por motivo de la pandemia.

Hasta 2007 la evolución del índice de cantidad de recurso trabajo empleado en la producción evoluciona de forma superpuesta prácticamente a la evolución del 


\section{FIGURA 2}

TASAS DE VARIACIÓN DEL OUTPUT, DE LOS INPUTS Y DE LA PRODUCTIVIDAD TOTAL DE LOS FACTORES, PTF: SNF EN ESPAÑA 2001-2019

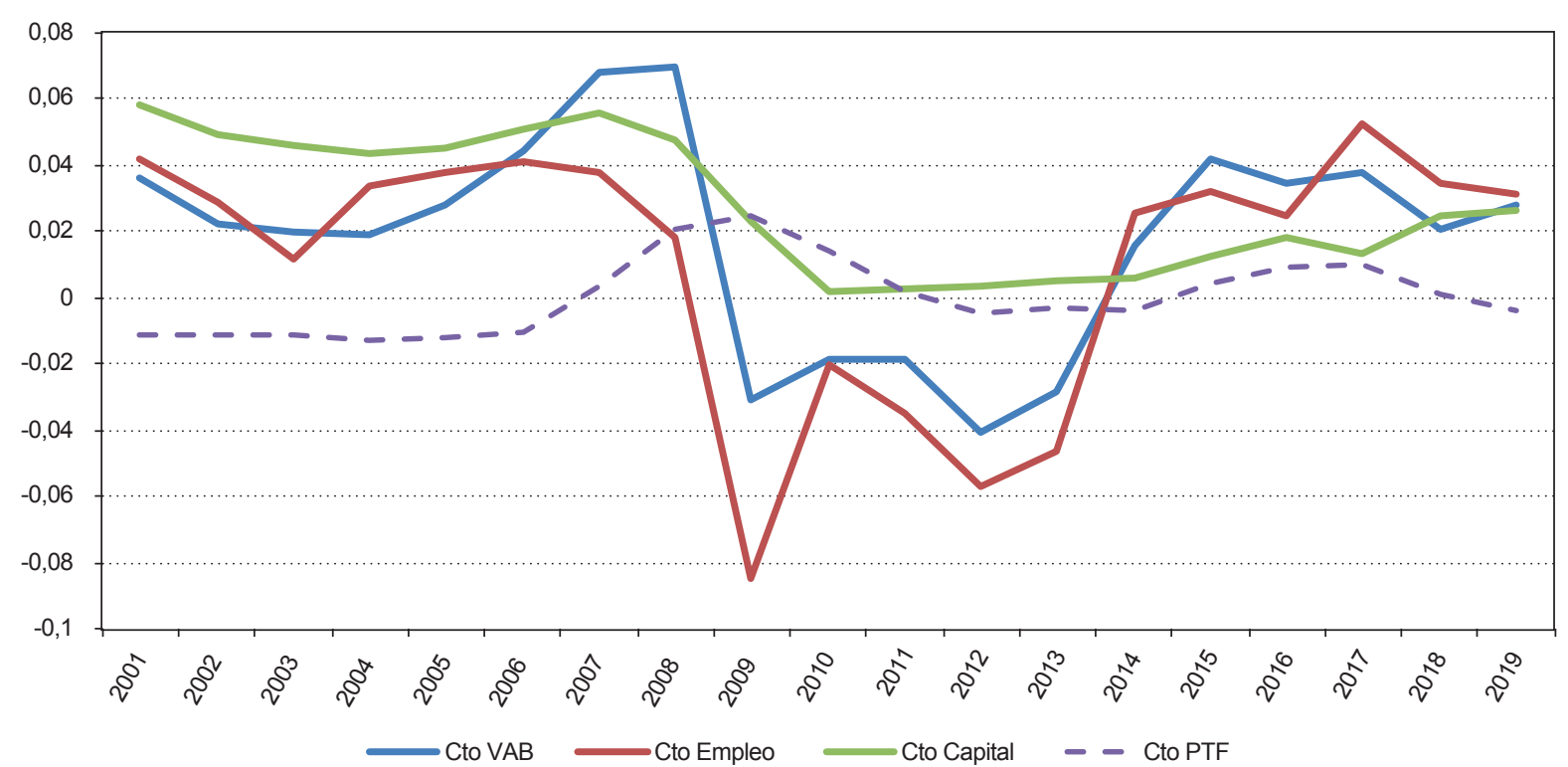

FUENTE: Elaboración propia a partir de los índices y datos de la Figura 1.

output mientras que la evolución del índice de servicios de capital evoluciona significativamente por encima: en 2008 el capital de las SNF para producir en España es un 50 \% mayor al del año 2000. En los años de la Gran Recesión, entre 2009 y 2013, la cantidad de input trabajo retrocede en una magnitud mayor a la contracción del output para situarse, en 2013, a niveles similares a los del año 2000. A partir de 2014 en adelante, la evolución del input trabajo ha sido paralela a la del output pero a un nivel inferior, de manera que el nivel de input máximo de 2008 no se alcanza hasta 2019. La cantidad de capital se estabiliza en los años de la Gran Recesión (la inversión nueva es casi igual a la amortización del capital existente) y, a partir de 2015, inicia una nueva expansión pero a tasas muy inferiores a las de principio del periodo (un crecimiento acumulado del $9 \%$ en cinco años).

La PTF se calcula como diferencia entre la tasa de crecimiento del output y la suma de las tasas de crecimiento de los input de trabajo y capital ponderadas, respectivamente, por 0,6 y 0,4, donde 0,6 es el promedio de la proporción de gastos de personal sobre el VAB para el conjunto del periodo y 0,4 es el complementario de 1; las cifras de tasa de crecimiento de la PTF que se representan en la Figura 2 son medias móviles de tres años.

La información de los índices en niveles de la Figura 1, se complementa con la información sobre tasas de variación interanual para cada uno de ellos, y sobre la tasa de crecimiento de la PTF (diferencia entre crecimiento del output y crecimiento de la suma ponderada de crecimiento de los inputs), que se presenta en la Figura 2. De la Figura 2 es evidente la aceleración en el crecimiento del output entre 2005 y 2007, impulsado por la elevada acumulación de capital que se produce desde el principio del periodo (con tasas medias de crecimiento anual por encima del $5 \%$ ). Las tasas 
negativas de crecimiento del input trabajo, superiores en valor absoluto a las de contracción del output en el periodo de la Gran Recesión (con la crisis financiera seguida de la crisis de la deuda pública de los países del euro, empezando por la deuda griega) explican el distanciamiento entre el índice del output y del trabajo en este mismo periodo, según la Figura 1.

La comparación de la tasa de crecimiento del output con la tasa de crecimiento de cada input permite conocer la evolución de la productividad parcial respectiva. Hasta 2006 la productividad del trabajo es prácticamente estable (crecimientos anuales similares), mientras que la del capital desciende porque el stock de este recurso crece más que el crecimiento del output. Entre 2007 y 2013 crece la productividad del trabajo, mientras que con la excepción de 2007 y 2008, la productividad del capital sigue en descenso. En la fase expansiva de 2014 en adelante, la productividad del trabajo se estanca y la del capital crece. En lo que se refiere al crecimiento de la PTF, este se mantiene estable con valores negativos alrededor del $-1,1 \%$ anual entre 2001 y 2006; en 2007-2010 el crecimiento pasa a valores positivos, con un crecimiento máximo del $2,4 \%$ en 2009. Coincide este crecimiento, por tanto, con la restructuración del sector empresarial en respuesta a la crisis financiera y la explosión de la burbuja inmobiliaria. A partir de 2014 en adelante, la PTF vuelve a crecer con una tasa media interanual del $0,45 \%$ entre 2014 y 20191.

\footnotetext{
1 Resulta de interés poner en perspectiva la evolución de la productividad que se desprende de la Figura 2 con valores de productividad disponibles para el conjunto de la economía española y para algunas economías del entorno en las estadísticas que publica la OCDE. Según estas estimaciones, en 2019 la productividad parcial del trabajo, en términos de PIB por hora trabajada en dólares de poder de paridad de compra constante, de España es un $73 \%$ de la productividad de Estados Unidos, algo por debajo del $80 \%$ de la productividad de Alemania y de Francia y muy cerca de la productividad de Italia. Por otra parte, el crecimiento anual de la PTF estimado para España con datos de OCDESTATA, es negativo para todo el periodo entre 2000 y 2011, con mínimos de $-0,27 \%$ en 2003 y 2004 . De 2012 en adelante, el crecimiento vuelve a valores positivos alrededor del 0,4\%. Entre 2000 y 2019 , la media de la variación anual de la PTF es 0,63 \% en Alemania, 0,39 \% en Francia, $0,79 \%$ en Estados Unidos, $-0,07 \%$ en España y $-0,24 \%$ en Italia.
}

\section{Tejido y demografía empresarial}

La dinámica en la evolución de la productividad está marcada por la creación destructiva que supone concentrar más actividad y más recursos bajo la gestión de empresas más eficientes en detrimento de la producción y recursos empleados de las menos productivas. La reasignación de recursos que permite hacer crecer la productividad media de la economía se produce por una doble vía: la rotación de empresas resultado del nacimiento de nuevas empresas y la muerte de otras que liberan recursos disponibles, y la ganancia de cuota de mercado por parte de las empresas más competitivas en detrimento de las menos competitivas. En este apartado se presentan algunas evidencias sobre la evolución en el número de sociedades no financieras en España (anónimas y limitadas), como un indicador más de la dinámica empresarial en los últimos veinte años ${ }^{2}$.

En el año 2000 el número total de sociedades en los sectores no financieros era de 696.670; de ellas, 551.839 con asalariados y 144.831 sin asalariados. En 2019 existen en España 1.181.879 sociedades mercantiles en los sectores no financieros de la economía española, casi un $70 \%$ más que las cerca de 700.000 del año 2000. En 2019 el 63,5\% de las sociedades tienen asalariados, cuando en el año 2000 esa proporción era del 80,3 \%. La Figura 3 muestra el índice de evolución temporal de las sociedades, total, con y sin asalariados, con base 1 en el año 2000. El número total de sociedades, así como el número de ellas con y $\sin$ asalariados, crece de forma importante hasta 2008 , de manera que en ese año hay un $53 \%$ $(133 \%)$ más de sociedades con ( $\sin$ ) asalariados que en el año 2000. Desde 2008 las sociedades sin asalariados siguen creciendo, aunque a tasas cada vez menores, mientras que las empresas con asalariados decrecen durante los años de Gran Recesión y se

\footnotetext{
2 Las sociedades mercantiles no financieras incluyen todas las sociedades del DIRCE excepto las de los sectores bancario, seguros, fondos (sectores CNAE 64 a 66). Ver García Perea (2020) para una exposición más completa de la demografía empresarial en España.
} 


\section{FIGURA 3}

\section{EVOLUCIÓN DEL NÚMERO DE SOCIEDADES EN LOS SECTORES NO FINANCIEROS EN ESPAÑA. ÍNDICE 1 EN 2000}

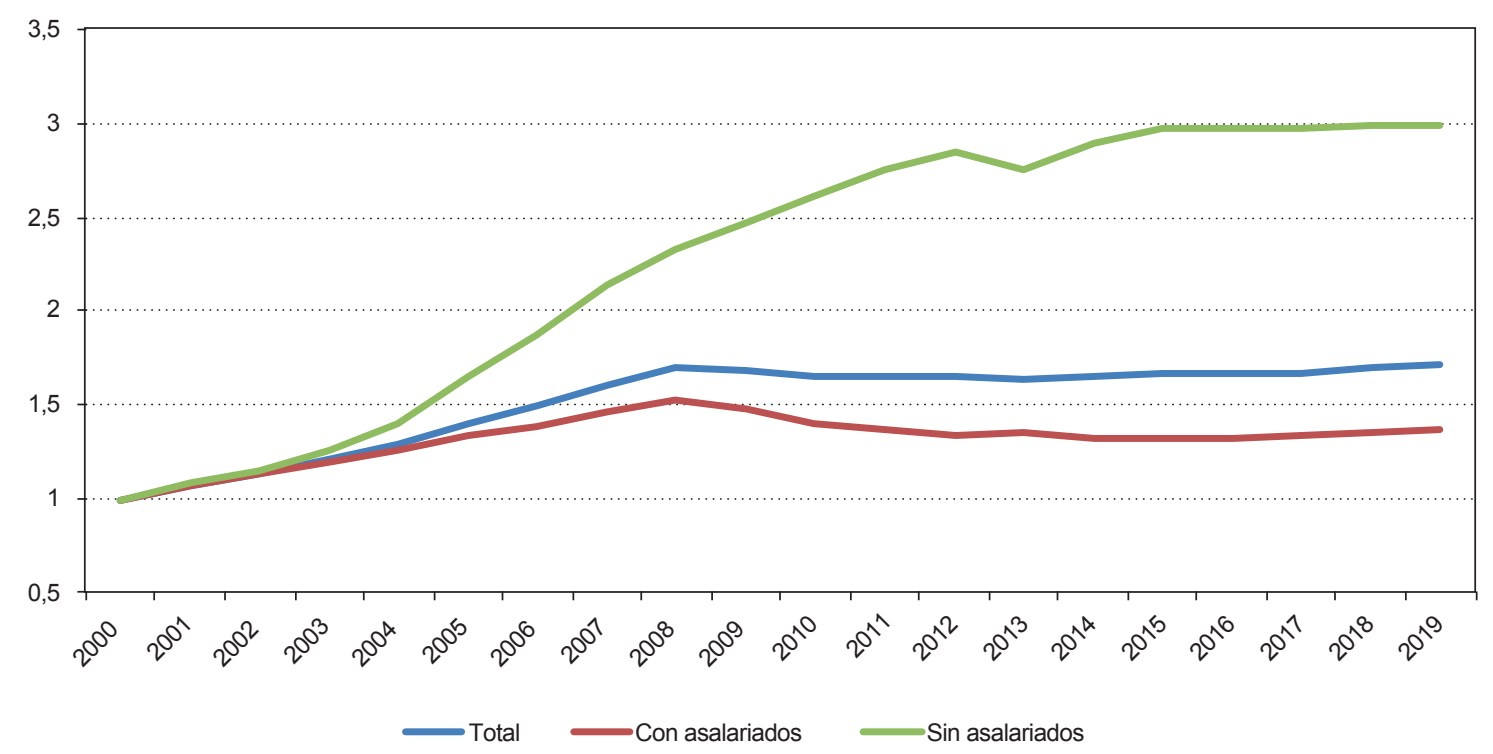

FUENTE: Elaboración propia a partir de los datos extraídos del Directorio Central de Empresas (DIRCE).

estabilizan en el periodo de recuperación a partir de 2014. Esto significa que en 2014 el número de sociedades con asalariados es un $14 \%$ inferior al de 2008.

Comparando la dinámica en el número de sociedades de la Figura 3 con la dinámica de output e inputs de la Figura 1 se comprueba el paralelismo entre las dos, especialmente con las sociedades con asalariados que razonablemente concentran prácticamente toda la actividad ${ }^{3}$. Durante los años de expansión previos a la crisis de 2008 , las sociedades con asalariados crecen más que el VAB real (6 \% frente a $4 \%$ de crecimiento medio anual, respectivamente), de manera que el output medio por empresa disminuye durante ese periodo. Durante la Gran Recesión la contracción en output y en número

\footnotetext{
3 EI DIRCE excluye a las empresas de los sectores agrícola y pesquero mientras que la contabilidad nacional de las SNF incluye la actividad de estos sectores.
}

de sociedades con asalariados es similar, alrededor de $12 \%$. Sin embargo, en los años de recuperación a partir de 2014, el output crece mientras el número de sociedades se estabiliza, lo que significa un crecimiento en el output medio por empresa. Estas dos circunstancias pueden tener efectos sobre la productividad. En primer lugar, la salida neta de empresas durante la Gran Recesión tendrá efectos positivos sobre la productividad si las empresas que permanecen son más productivas que las que desaparecen. En segundo lugar, aumentos en el tamaño medio de las empresas aumentan la productividad en presencia de economías de escala.

La evolución de las sociedades en España tiene un importante componente sectorial, con diferencias sustanciales entre la evolución del número de sociedades en la industria, en los servicios y en la construcción. En el sector industrial, el número de sociedades se mantiene relativamente estable en todo el periodo 
con 110.706 unidades en el año 2000 y 117.245 en el año 2019; el número máximo de sociedades industriales es 133.276 en 2008, un $20 \%$ mayor que en el número de sociedades en el año 2000. La evolución del número de sociedades muestra su mayor volatilidad en el sector de la construcción con un aumento del $75 \%$ en el número de sociedades en 2008 con respecto a 2000 (291.669 sociedades frente a 166.422), y una reducción del 31 \% (hasta 200.875) en 2019. En el sector servicios, el número de sociedades crece durante todo el periodo, aunque el ritmo de crecimiento es similar al del sector de la construcción hasta 2008 y mucho más lento en los años sucesivos. Para explicar la elevada dinámica empresarial en el número de sociedades entre 2000 y 2008 , hay que tener muy en cuenta el hecho singular de la fuerte expansión de los sectores de la construcción y afines.

Dentro del conjunto de las sociedades con asalariados resulta de interés conocer la distribución por tamaños y cómo varía en el tiempo, por sus implicaciones para la evolución de la productividad. Entre las empresas de menor dimensión se producirán las mayores tasas de natalidad y mortalidad empresarial pero las empresas que sobrevivan van a tener un mayor potencial de crecimiento. Por otra parte, se sabe también que la productividad media del trabajo crece con el tamaño de las empresas. Idealmente, por tanto, la dinámica empresarial que asegura que una economía es más productiva que otra y que esa diferencia se mantiene o incluso aumenta en el tiempo, requiere dos condiciones: que en la situación estacionaria, la primera tenga un tamaño medio de las empresas mayor que la segunda, y que la primera tenga mayor rotación empresarial por entradas y salidas de empresas, incluidas las creaciones y cierres, en todas las clases de tamaño (Haltiwanger et al., 2017; Decker et al., 2016; Bijnens \& Koninigs, 2020).

La Figura 4 muestra las tasas de variación interanual en el número de sociedades con entre uno y cinco asalariados, y la variación en el número de sociedades con seis o más trabajadores. En los años expansivos, entre 2000 y 2008 , los dos colectivos crecen a tasas interanuales alrededor del $6 \%$, lo que deja intacta la distribución de sociedades entre las dos clases de tamaño, $72 \%$ para la de entre uno y cinco trabajadores y $28 \%$ para las de seis o más. Durante la Gran Recesión el número de sociedades disminuye en los dos colectivos pero sensiblemente más en el de empresas con seis o más trabajadores. A ello contribuirán tanto el cierre de empresas como consecuencia de la caída de la demanda como la disminución de empleo entre las supervivientes, que supondrá pasar a una clase de tamaño inferior. En la recuperación a partir de 2014, el número de empresas con entre uno y cinco trabajadores se estanca, mientras que el de más de seis aumenta tasas por encima del $3 \%$. Podría afirmarse, por tanto, que en la reciente recuperación la entrada de sociedades en el colectivo de entre uno y cinco trabajadores, principalmente por nuevas creaciones, es similar a la tasa de cierre más la tasa de empresas que pasan a clases de tamaños superiores ${ }^{4}$.

Se confirma así una vez más que, en la recuperación a partir de 2014, el dinamismo empresarial se manifiesta más por el crecimiento de las empresas existentes que por la renovación del tejido empresarial. Esto podría resultar positivo para el crecimiento de la productividad porque aumenta el tamaño medio de las empresas, pero también puede darse el efecto negativo sobre el crecimiento de la productividad por falta de renovación del tejido productivo ${ }^{5}$.

\footnotetext{
4 EI DIRCE distingue entre empresas con 6-9 trabajadores, con $10-19$ y con 20 o más trabajadores. El crecimiento en el número de sociedades, a partir de 2014, aumenta al pasar a clases de tamaños mayores: $13 \%$ de aumento entre 2014 y 2019 para las de 6-9 trabajadores, $19 \%$ para las de $10-19$ y $31,4 \%$ para las de 20 o más.

5 EI DIRCE publica las tasas de creación y destrucción de empresas para todo el colectivo, sin diferenciar por forma jurídica; Eurostat, por su parte, ofrece información sobre tasas de nacimiento y muerte de empresas que permiten comparaciones entre países. Los datos ponen de manifiesto un descenso importante en las tasas de creación y nacimiento después de 2008 con respecto a los de años anteriores, si bien, como se ha señalado antes, las cifras de creación de empresas hasta 2008 estarán muy distorsionadas por la expansión atípica del sector de la construcción. Las comparaciones de España con otros países en nacimiento y muerte de empresas ponen de manifiesto mayores tasas de muerte de empresas que en los países próximos en la Gran Recesión, pero también una mayor tasa de nacimiento en los años de recuperación (García Perea, 2020).
} 


\section{FIGURA 4}

\section{TASAS DE VARIACIÓN EN EL NÚMERO DE SOCIEDADES CON ASALARIADOS PARA DOS CLASES DE TAMAÑOS, ENTRE 1 Y 5 TRABAJADORES Y 6 O MÁS TRABAJADORES}

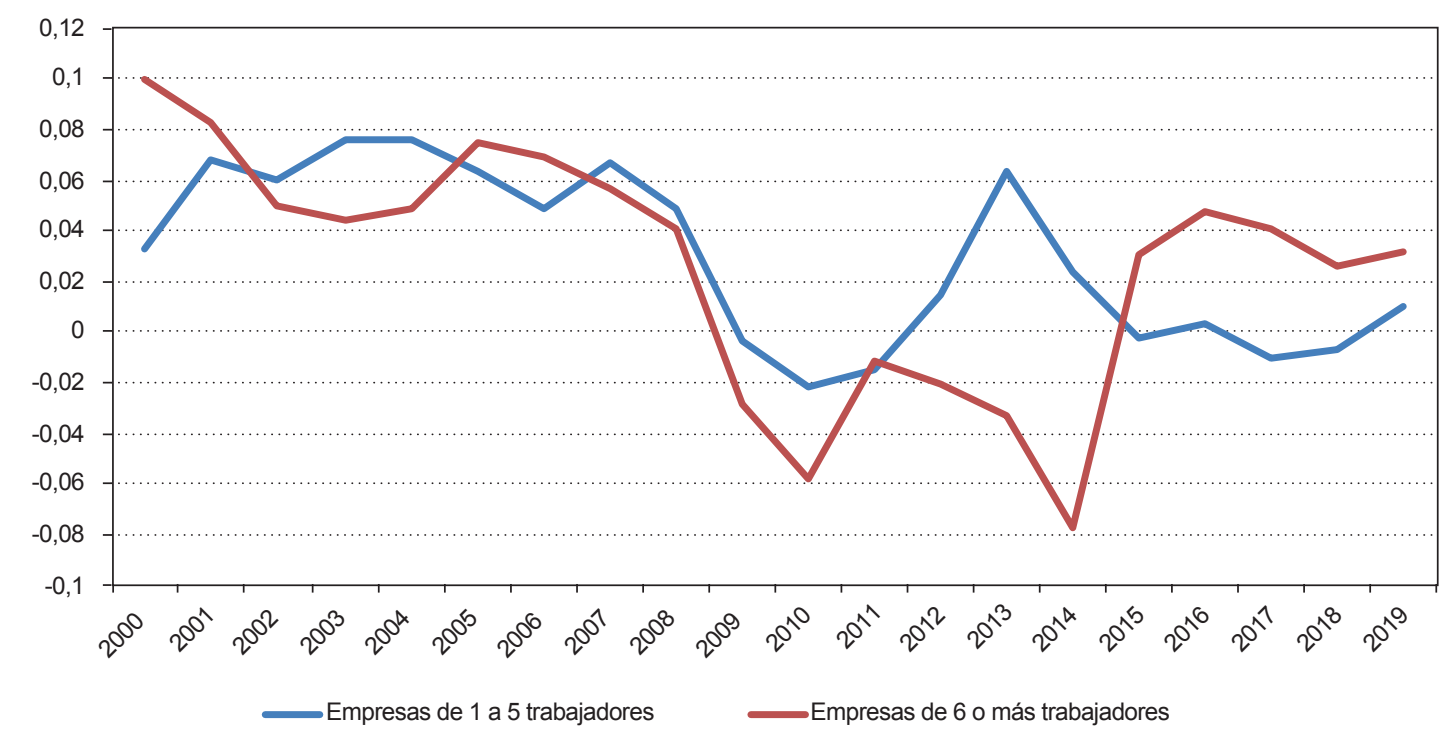

FUENTE: Elaboración propia a partir de datos DIRCE.

3. Factores internos de competitividad de la empresa

La competencia en los mercados disciplina la conducta de las empresas y recompensa a las organizaciones más productivas permitiéndoles gestionar mayores volúmenes de recursos en detrimento de las menos competitivas que, o bien son expulsadas del mercado o ven reducida su cuota (Foster et al., 2006). En economías abiertas, la competencia tiene un componente exterior de manera que la producción nacional aumenta o disminuye como resultado de las exportaciones y las importaciones. La política industrial trata de potenciar la competitividad de las empresas que producen en el territorio nacional, para un nivel de competencia en el mercado, mientras que la política de defensa de la competencia trata de mantener los estímulos externos para que las empresas produzcan más y vendan la producción a precios más bajos.
Esta visión de la política industrial y de la política de defensa de la competencia tiende a contemplar la empresa como una unidad de producción donde toda la información sobre la misma se resume en la tecnología sobre las posibilidades de producción en un momento del tiempo. Lo que ocurre dentro de la empresa, cómo se organiza y cómo se gestiona, por qué utiliza recursos de una naturaleza u otra, por qué unas empresas innovan más que otras, se ignora totalmente y con ello se pierde capacidad explicativa sobre la creciente heterogeneidad empresarial (Syverson, 2011; Autor et al., 2020). Y esta falta de reconocimiento de la diversidad empresarial plantea dudas sobre la capacidad disciplinaria y homogeneizadora de la competencia. Es por ello que la política industrial y la política de competencia necesitan conocer mejor el funcionamiento interno de las empresas como primer paso para comprender la persistencia de la heterogeneidad empresarial 
y sus consecuencias. En este apartado se presentan algunos de los rasgos distintivos que definen las características internas de las empresas españolas, extraídos de algunas investigaciones recientes (Huerta \& Moral, 2018; Huerta \& Salas, 2014, 2017, 2018; Mas, 2020; European Company Survey, 2019; Myro, 2018; Bayo \& Ortín, 2018; Garcés-Galdeano et al., 2016; GarcésGaldeano \& Huerta, 2019), que pueden explicar por qué la productividad media de la economía española diverge de la productividad media de los países frontera.

Uno de los rasgos que definen el capitalismo del siglo XXI es la creciente importancia de los activos intangibles (calidad de gestión, software, conocimiento, personas, organización, reputación...) en el total del capital productivo de las empresas, y el correspondiente menor peso de los tangibles (maquinaria, instalaciones, inmuebles). La intangibilidad de los recursos productivos y las diferentes capacidades constituye una fuente de heterogeneidad empresarial; se ha demostrado que existe una fuerte complementariedad entre ellos que no todas las empresas saben cómo aprovechar mejor, los intangibles, además resultan de difícil imitación por su invisibilidad y falta de estandarización (Haskel \& Westlake, 2018). Por otra parte, distintos estudios han puesto de manifiesto la estrecha correlación positiva entre dotación de intangibles en el conjunto de las economías, y diferencias en productividad (Corrado et al., 2016). Mas (2020) analiza la dotación de activos tangibles e intangibles en la economía y las empresas españolas, desde una perspectiva de comparación internacional. Utilizando información procedente de la base de datos EUKLEMS, el estudio documenta que España es un país homologable a la mayoría del resto de países desarrollados en inversión en activos tangibles, pero ocupa una de las últimas posiciones dentro del grupo de 14 países de la UE en dotación de activos intangibles. Además, en el caso español, la inversión en intangibles está orientada hacia actividades que tienen un menor impacto sobre las mejoras de productividad a medio y largo plazo. En los países más productivos de la UE el peso mayoritario de la inversión recae en los ámbitos de la I+D, software y la destinada a mejorar la estructura organizativa de las empresas, precisamente los intangibles en los que la inversión en España es menor.

La relevancia de las capacidades organizativas de las empresas y de la calidad de la gestión para la productividad vuelve a ponerse de manifiesto en el reciente estudio de Schivardi y Schmitz (2019). La investigación constata que desde mediados de los años noventa, el crecimiento de la productividad ha sido sustancialmente más reducido en los países del sur de Europa que en los del norte. Se argumenta que esta divergencia tiene su origen parcialmente en ineficiencias en las prácticas de gestión empresarial en los países del sur, de manera que impiden a sus empresas aprovechar las mejoras potenciales con la incorporación de las TIC (tecnologías de la información y la comunicación). Las TIC generan divergencia y aumentan las diferencias de productividad entre los países por tres razones. Primera, porque existen diferencias en la intensidad de inversión en TIC entre países; segunda, porque existen diferencias entre países en la calidad de gestión de las empresas, lo que causa diferencias en la traslación a aumentos en la productividad de la misma inversión en TIC; y tercera, porque la dinámica digital fomenta que los mejores salarios que se ofrecen en los países más eficientes y que mejor utilizan las tecnologías digitales, resulten más atractivos para los trabajadores cualificados de los países del sur de Europa que emigran hacia los países con salarios más altos aumentando las diferencias en dotación de capital humano. Todo ello se traduce cuantitativamente en el resultado final: la gestión ineficiente de las empresas, su impacto en la inversión en TIC y la dotación de capital humano explican el $35 \%$ de la divergencia de productividad de Italia, el $47 \%$ de España y el $81 \%$ de Portugal con respecto a la productividad de Alemania en el periodo 1995/20176.

\footnotetext{
6 CES, Consejo Económico y Social de España (2020) y Cuadrado et al. (2020) ofrecen resultados en la misma dirección demostrando la importancia de las prácticas de gestión y de la formación de los trabajadores para el óptimo aprovechamiento de las TIC en la mejora de la productividad.
} 
Además del World Management Survey de donde procede la información sobre calidad de gestión que se utiliza en el análisis de Schivardi y Schmitz (2019), otros estudios han profundizado en la evaluación de las prácticas de gestión extendidas entre las empresas españolas, así como en las dotaciones de capital organizacional que igualmente se han demostrado complementarias con las inversiones en TIC (Rubio et al., 2018; Garcés-Galdeano et al., 2016; GarcésGaldeano \& Huerta, 2019). En estos estudios la calidad de gestión y las capacidades organizacionales sobre las que se apoya, incluyen: i) la capacidad para supervisar, evaluar el desempeño y de mejorar los procesos existentes; ii) capacidad para fijar objetivos adecuados, a respetarlos y a modificar la estrategia en caso de incoherencia con los objetivos; y iii) capacidad para potenciar el trabajo de las personas a través de la implantación de sistemas de incentivos al desempeño y a la promoción del talento.

\section{Las capacidades organizativas y de gestión de las empresas en España}

La evidencia sobre la gestión del capital humano en las empresas españolas pone de manifiesto con gran claridad el escaso valor estratégico que se le asigna al capital humano, limitándose a una gestión de personas únicamente defensiva. Bayo y Ortín (2018), utilizando información de la Fundación Europea para la Calidad de las Condiciones de Vida y de Trabajo y a través de una treintena de indicadores de gestión de las personas, han comparado la utilización de prácticas de recursos humanos de alto rendimiento en las empresas españolas con la utilización por parte de las empresas en el resto de países de la UE. En la utilización de prácticas avanzadas de gestión de personas, España se sitúa en el puesto 21 de 28 países de la UE, cerca de Rumanía, Bulgaria, Croacia e Italia, y lejos de países de referencia como Finlandia, Dinamarca o Suecia. De las prácticas estudiadas, aquellas en las que hay un notable margen de mejora son las relacionadas con la seguridad en el empleo, las perspectivas de desarrollo de carrera profesional, la formación en el trabajo y las políticas retributivas.

Las relaciones de confianza y colaboración entre la dirección y los empleados dentro de la empresa son un elemento capital del compromiso de las personas con el proyecto empresarial. Así, de acuerdo con la información proporcionada por la European Company Survey (ECS, 2019), la opinión de los managers respecto al nivel de motivación de los empleados, es la siguiente: en el conjunto de la UE, el $22 \%$ de los managers encuestados considera que los trabajadores de su empresa están poco o muy poco motivados. Este porcentaje alcanza un $38 \%$ en España, mientras que en Francia o Alemania es del $16 \%$. En cuanto al clima laboral, el $79 \%$ de los managers consideran, en el conjunto de la industria en la UE, que mantienen una buena o muy buena relación con los empleados. En España esa consideración baja al $63 \%$ alejada de países como Alemania o Francia donde estos porcentajes alcanzan el $78 \%$ y $86 \%$, respectivamente.

Esta visión de los managers respecto a los trabajadores y la confianza recíproca que tienen entre sí, se refleja también en las dificultades para compartir información y actuar de manera trasparente en la organización. Así, cuando se pregunta a los directivos sobre si realizan regularmente reuniones con todos los trabajadores para explicar la organización del trabajo, en España las realizan solo el $19 \%$ de las empresas, en la UE el $28 \%$, en Francia el $26 \%$ y en Alemania el $29 \%$. De forma similar, cuando se pregunta a las empresas sobre si utilizan distintos sistemas (emails, newsletters, redes de comunicación) para diseminar y compartir la información con los trabajadores, en España contestan que lo hacen regularmente el $28 \%$, en la UE el $34 \%$, en Francia el $50 \%$ y en Alemania el $32 \%$. En cuanto a la discrecionalidad que se da a los trabajadores para organizar sus tareas, se observan notables diferencias entre países. Mientras 
que en España los managers afirman mayoritariamente que menos del $20 \%$ de la plantilla tiene la posibilidad de organizar su horario y trabajo, en el conjunto de la UE son mucho más frecuentes las empresas en las que un elevado porcentaje de trabajadores tienen autonomía para organizar sus tareas. Estudiando conjuntamente la complejidad de las tareas y la autonomía, se observa que en España está muy extendido el mando y control frente a otras opciones que implican más responsabilidad y autonomía individual. Así, en España el $63 \%$ de los managers reconocen que predomina el mando y control frente a un $48 \%$ en la UE, un $42 \%$ en Francia y un $39 \%$ en Alemania.

\section{La calidad del recurso empresarial}

La realidad que observamos sobre la calidad del recurso empresarial es bastante heterogénea. La evidencia para la empresa española indica, que si bien a nivel de actividad emprendedora, España se sitúa en niveles similares a los países de nuestro entorno, cuando ponemos el foco en sectores de alto potencial de crecimiento y tecnológicamente avanzados, es notablemente menor que la de países como Francia, Reino Unido, Alemania o EE UU (De Castro et al., 2018). De manera complementaria, la evidencia apunta a posibles deficiencias en el mercado de directivos y empresarios en la asignación del talento empresarial (Huerta \& Salas, 2018). Así la productividad media de las empresas españolas no solo es menor que la de las empresas alemanas en las distintas clases de tamaño, sino que las diferencias de productividad entre las empresas más pequeñas y las más grandes es del $20 \%$ en Alemania, mientras que supera el $40 \%$ en España. La fragmentación y el reducido tamaño de las empresas españolas con un predominio de las microempresas y pequeñas empresas y la baja productividad de todas ellas se asocia a factores múltiples, pero también influye la limitada ambición de muchos proyectos empresariales, la baja cualificación y formación de muchos empresarios españoles, la baja cualificación de operarios, la poca delegación en su forma de dirigir y el predominio del papel del jefe como controlador frente al de facilitador de iniciativas y proyectos. A la pregunta en la mencionada encuesta ECS de cómo describirían los managers el liderazgo que se establece en su empresa, mientras que en Alemania o Francia un $77 \%$ de los directivos se ven como facilitadores del trabajo y un $23 \%$ como controladores, en nuestro país los porcentajes son un $64 \%$ y un $36 \%$, respectivamente.

Rubio et al. (2018), a través de un índice de buenas prácticas de dirección a partir de cuarenta variables descritas en la Encuesta de estrategias empresariales de empresas españolas (ESEE), encuentran una relación positiva y significativa estadísticamente entre la adopción de buenas prácticas de dirección y la cifra de ventas alcanzada, así como la cuota de mercado obtenida. Se observa, por tanto, que las empresas mejor dirigidas no solo consiguen mayores cifras de ventas, sino también tienen mejores posiciones competitivas en sus mercados. Los resultados revelan una productividad superior de las empresas con mayores niveles de intensidad en el uso de buenas prácticas directivas.

En relación a las capacidades directivas en las pymes, Garcés-Galdeano y García-Olaverri (2019), basándose en la información de una encuesta llevada a cabo a 1.500 gerentes de empresas de menos de 50 trabajadores operando en sectores de alta y mediaalta tecnología, observan las siguientes evidencias: en cuanto a la formación, el $31 \%$ de los directivos carece de estudios universitarios. Tan solo el $16 \%$ de ellos tiene estudios de Máster o Doctorado. De los que tienen estudios universitarios, tres cuartas partes tienen algún tipo de formación en administración o gestión de empresas, mientras el $25 \%$ restante no tiene conocimiento en esos ámbitos. Esos porcentajes son del $42 \%$ y $58 \%$, respectivamente, entre los que no tienen formación universitaria. En relación con la experiencia, el $43 \%$ de los managers no ha tenido ninguna experiencia directiva en otra compañía y mayoritariamente acumulan toda su vida laboral (no necesariamente en la posición de manager) en el mismo sector de actividad. Con una 
edad promedio de 45 años y más de 19 años de experiencia en el mismo sector industrial, emerge un perfil directivo de un manager que apenas ha tenido experiencia laboral fuera de su sector e incluso fuera de la empresa que dirige. Atendiendo a la actividad emprendedora, más del $90 \%$ de los directivos entrevistados no ha participado nunca en la creación de una empresa. Tan solo el $3 \%$ ha tenido una actividad emprendedora destacable participando en la creación de tres o más compañías.

A grandes rasgos se observan dos grupos bien diferenciados de directivos: por un lado, los que tienen un conocimiento profundo del negocio, basado en su amplia experiencia en el sector y en la empresa que dirigen y no tanto en su formación; a este grupo pertenece casi el $70 \%$ de los entrevistados. Por otro lado, los que aportan un conocimiento más variado, con experiencia directiva en otras compañías o alguna iniciativa emprendedora; características que van ligadas a una mejor formación, tanto general como en el ámbito de la administración de empresas. En este colectivo estaría el $30 \%$ de los entrevistados.

El hecho de que el colectivo de empresas estudiado pertenezca a sectores tecnológicamente avanzados parecería requerir de unos directivos mejor formados en herramientas y técnicas de gestión, con más movilidad de los directivos entre empresas y mayor actividad emprendedora. La realidad es distinta: la experiencia en una única empresa y una escasa actitud emprendedora, hacen pensar en un cierto conformismo y limitadas ambiciones en la definición y construcción del proyecto empresarial.

En resumen, de la exposición previa se desprende, primero, que los activos intangibles se están consolidando como las principales fuentes de crecimiento $y$ productividad de las economías y empresas más avanzadas; segundo, que las empresas son depositarias de gran parte de esos activos, que deben gestionarse teniendo en cuenta la fuerte complementariedad entre personas, conocimiento, calidad de gestión y competencias organizacionales; y tercero, las empresas españolas muestran déficits importantes en activos intangibles cuando se comparan con las dotaciones de estos recursos entre las empresas de los países más productivos de la UE. Estas consideraciones deberían ser el punto de partida de la política industrial del siglo $\mathrm{XXI}$, lo que significa colocar a la empresa y la innovación en el mismo plano de importancia, al menos, que el mercado como centro del análisis y foco de las políticas públicas de corte microeconómico.

\section{La estrategia de innovación de la UE}

Desde hace unos años, la Comisión Europea ha trabajado en la evaluación de las iniciativas y programas de I+D+i; además, ha elaborado un conjunto de recomendaciones estratégicas que han de guiar el futuro del programa marco europeo en materia de políticas de innovación. Tres han sido los objetivos fijados: innovación inteligente, inclusión y sostenibilidad. Se considera (Mazzucato, 2018) que la investigación y el desarrollo $(I+D)$ debe ser el pilar clave de la estrategia europea para 2030, para cuya instrumentación será fundamental repensar las políticas públicas de incentivos a la I+D y proveer de estímulos para cocrear y codiseñar nuevas empresas, tecnologías y mercados.

La Unión Europea lleva décadas de retraso, tanto en los resultados de innovación como en cifras de inversión en I+D, en comparación con Japón o EE UU.

Por lo tanto, la política de innovación del pasado debe revisarse en profundidad, no solo en términos de objetivos de inversión en I+D en relación al PIB, sino también en los objetivos y las características de los proyectos a impulsar.

De retos a misiones: en este contexto, políticas orientadas a misiones pueden ayudar a crear nuevas oportunidades de innovación y, a la vez, estimular las inversiones privadas en I+D. La idea básica es orientar la inversión privada en I+D a misiones específicas con objetivos claros y alcanzables fijados por la Administración en consonancia con la comunidad científica pública y privada. 
La UE considera que una misión establece un marco de oportunidad para encontrar soluciones a problemas. La gestión de cada misión requiere según la UE:

- Objetivos con resultados medibles y alcanzables en un plazo determinado. Por ejemplo, reducción de las emisiones en un $30 \%$ para 2030.

- Tener impacto a nivel social y en las decisiones políticas.

- Utilizar los ODS (objetivos de desarrollo sostenible) como fuente de su diseño e implementación.

- Obtener una amplia gama y cantidad de recursos públicos y privados.

- Estimular la colaboración entre disciplinas y tecnologías.

- Estar abiertos a propuestas y soluciones de abajo a arriba.

- Aprovechar las sinergias de otros programas de la UE, así como los ecosistemas de innovación nacionales y regionales.

En suma, la nueva orientación de la política de innovación de la UE, y también de España como país miembro, resulta interesante y novedosa porque traslada los objetivos de crecimiento económico desde la riqueza al bienestar de las personas, reconociendo que las medidas habituales de productividad o renta per cápita no son suficientes como indicadores de la calidad de vida. Plantea que los ODS, en cuanto que mejores indicadores del bienestar social que el PIB per cápita, se conviertan en los objetivos centrales de las políticas públicas en general. La política de innovación europea aspira a que las empresas interioricen los objetivos de desarrollo sostenible en sus decisiones de gestión, y espera que los incentivos públicos sirvan para alinear intereses particulares y generales.

Estando de acuerdo en que la política industrial, en general, y la de innovación, en particular, del siglo XXI, debe alinearse con los objetivos de bienestar social en sus misiones e instrumentos, creemos también que la articulación de las políticas públicas alrededor de misiones activas resulta todavía insuficiente para los objetivos últimos que se desean conseguir. En primer lugar, porque, en nuestra opinión, la eficiencia y la productividad, es decir la capacidad de crear riqueza, no debe perder peso entre los objetivos de las políticas públicas; lo que debe hacerse es reconocer la pluralidad de resultados que definen el bienestar colectivo, eficiencia, sostenibilidad e inclusión y avanzar en los tres de forma conjunta. En definitiva, ampliar los indicadores de eficiencia para tener en cuenta los efectos externos de las decisiones individuales. En segundo lugar, porque la misión activa mantiene la tradicional visión de la empresa como caja negra, con el beneficio privado como único motor de la conducta empresarial; las políticas públicas de corte microeconómico deben de tomar en consideración las corrientes de cambio en el ámbito empresarial que equiparan el propósito social al beneficio como guías de las decisiones de asignación de recursos (Mayer, 2020). En tercer lugar, y relacionado con lo anterior, se ignora la complementariedad entre intangibles y se ignoran la calidad de gestión y las competencias de organizaciones como determinantes de la performance empresarial, es decir, se sigue menospreciando el cómo las empresas consiguen los resultados individuales y colectivos.

\section{Propuestas de estrategias industriales}

Tres vías fundamentales se proponen para actuar sobre la situación descrita de las empresas en España. La primera, enfatiza la importancia de abrir la caja negra de la empresa para mejorar la calidad de la gestión mediante la difusión y extensión de buenas prácticas empresariales; la segunda, complementaria de la anterior, reconoce la importancia de la competencia en los mercados como medio para estimular el cambio y favorecer la sofisticación de los negocios. La tercera, se refiere al desarrollo de estrategias industriales centradas en la generación de ecosistemas que faciliten la inversión en activos intangibles y el aprovechamiento de efectos conjuntos que se centren en la innovación. 


\section{La importancia del management: política empresarial}

Hay que mejorar la dotación de capital intangible en las empresas españolas y difundir y extender un conjunto de prácticas de gestión de excelencia entre las organizaciones que están dentro de la frontera de productividad. Existen ejemplos de empresas españolas que son líderes mundiales en sus nichos de negocio. Si tenemos buenos ejemplos de modelos empresariales exitosos, resultaría importante que esas experiencias y prácticas se difundieran con mayor rapidez para que se extendieran a los otros colectivos empresariales que todavía o no las conocen o tienen dificultades para aplicarlas. Las iniciativas y prácticas adoptadas varían entre empresas y las soluciones tienen que ser sensibles a las particulares características del sector, tamaño y naturaleza de la competencia en sus mercados.

Avanzar en esa dirección no va a resultar sencillo. La introducción de nuevos modelos de empresa requiere, en la mayoría de los casos, del desarrollo de nuevas capacidades y competencias organizacionales y comporta un excelente liderazgo y un buen management. Por ello tres programas son importantes:

1) Profesionalizar la gestión empresarial: en un marco institucional de libre mercado una vía habitual de acceso a puestos de alta dirección empresarial es por la propiedad directa o indirecta del capital empleado en la producción. Las personas con una elevada riqueza personal cuentan con suficientes recursos propios para crear una empresa y asumir su dirección si así lo desean, estén o no cualificados para ello. Aunque los resultados económicos negativos erosionen el capital y la riqueza personal, el empresario puede decidir prolongar la continuidad de la empresa más allá de lo que la eficiencia social recomendaría, por otros intereses personales. Profesionalizar la gestión, es un camino para mejorar las habilidades de quienes dirigen la asignación de recursos. España tiene un largo recorrido para converger a los niveles de profesionalización de la gestión de países de su entorno especialmente en el ámbito de las empresas familiares.
2) Reducir los costes organizativos de crecer: la dirección marca el rumbo de los proyectos empresariales y define un modelo de gestión en la que la forma de organizar la colaboración de los especialistas en la ejecución de estrategia adquiere una especial relevancia. La organización amplifica, más o menos, el impacto de las decisiones estratégicas en los resultados empresariales y, por ello, es también una dimensión crítica para reducir los costes al crecimiento. Como regla general, un modelo organizativo descentralizado libera a la dirección de tiempo dedicado a coordinar y controlar las decisiones de personas y recursos bajo su autoridad y, en consecuencia, permite dirigir un volumen de recursos mayor. En resumen, la delegación eficiente reduce los costes internos de crecer en las organizaciones.

3) Avanzar hacia un proyecto empresarial de ganancias compartidas: en la empresa española, como hemos visto, el nivel de confianza entre los protagonistas de la acción colectiva es bajo y el grado de delegación de poder de decisión en las organizaciones también. Converger en niveles de confianza con los países de mayor productividad y tamaño empresarial es otra de las vías de avance hacia empresas más grandes y productivas. Una buena medida para aumentar la confianza sería incorporar incentivos basados en la participación financiera de los empleados en los resultados de la organización. Es decir, compartir los resultados de la acción de la empresa.

Las asociaciones empresariales, clusters y agrupaciones sectoriales, así como las agencias públicas de innovación y desarrollo deben ejercer un papel relevante en las iniciativas de debate y extensión de buenas prácticas para la mejora de la gestión empresarial.

\section{Un nuevo papel para la política de defensa de la competencia}

La política de competencia en los distintos países de la UE se enfrenta a retos importantes que dificultan su papel e influencia sobre las empresas y los mercados. 
Los desafíos aparecen como consecuencia del cambio tecnológico y las economías de red que se están generando en algunos sectores; el peso creciente de los intangibles en la ventaja competitiva de las empresas (más difíciles de imitar) y el proceso de globalización y cambio de los mercados de referencia donde compiten las empresas, hacen que la política que aisladamente desarrolle un país sea poco eficaz. En este contexto, promover la importancia de la eficiencia dinámica como objetivo central de la política de la competencia puede resultar valioso. Se trata de reconocer que si hay innovación, el beneficio extraordinario de hoy se justifica y se compensa cuando se ofrece más innovación y así aumenta el bienestar de los consumidores en el futuro, al ampliar la gama de nuevos productos que generan un valor creciente.

El papel fundamental de la competencia debe ser contribuir a mejorar la prosperidad de un país y, por tanto, incrementar el bienestar de los consumidores a lo largo del tiempo mediante el impulso a la rivalidad que fomente la innovación y la mejora de la productividad de las empresas. La innovación en las empresas requiere de tecnología, conocimiento, compromiso de las personas, visión a largo plazo, inversión sostenida en intangibles y entornos propicios a la extensión y difusión de buenas prácticas de gestión. Schumpeter (1939) y Stiglitz (1994) sugieren de forma convincente que estructuras de mercado intermedias, ni muchas empresas pequeñas ni una muy grande, impulsan con frecuencia el avance de la tecnología ya que permiten a las empresas obtener unos beneficios aceptables, invertir en intangibles y así desplazar la frontera de la innovación. Además, la competencia mediante la diferenciación e innovación (Porter, 1985) es una fuente de ventaja competitiva más sostenible para las empresas y más interesante y valiosa para los consumidores que la centrada en la imitación y precios.

En definitiva, la política de la competencia, manteniendo el principio fundamental de que la competencia en los mercados es una fuente de estímulo y dinamismo esencial para las empresas y la economía, debe aplicarse considerando que el objetivo central es el de garantizar la eficiencia dinámica de los mercados $y$, por tanto, tiene que estimular la innovación de las empresas y la mejora de su capacidad para generar más y más valor para los consumidores finales. Por un lado, esta visión de la competencia significa considerar el difícil equilibrio que se produce y que habrá que evaluar caso a caso, entre la eficiencia dinámica (innovación y beneficios extraordinarios) y la eficiencia estática (precios igual a coste marginal y márgenes bajos). Por otro lado, el debate de la política de la competencia deja fuera de su intervención cómo se pueden alcanzar objetivos socialmente deseables relacionados con la sostenibilidad de los resultados y su equidad, por lo que esta política es complementaria y no sustituye a otras iniciativas de política industrial.

\section{Una nueva estrategia para impulsar la innovación: crear y desarrollar ecosistemas}

El debate de la política industrial se ha desplazado (Myro, 2018; Warwick, 2013) de una visión tradicional que se desarrolló en los ochenta del siglo pasado basada en subsidios, empresas públicas, apoyo a campeones nacionales, ayudas a empresas en crisis y políticas tarifarias hacia las recientes dinámicas centradas en los fallos del mercado y su corrección mediante impuestos y subsidios, operando en los mercados de factores (incentivos a la I+D+i, ayudas al empleo, incentivos fiscales a la inversión o ayudas a la financiación de actividades de alto riesgo). Recientemente se ha producido un nuevo cambio, y la política industrial se orienta a la construcción de sistemas y redes y hacia la creación de instituciones que ayuden a alinear los intereses y objetivos de los protagonistas del espacio económico y faciliten la inversión en activos intangibles.

Las nuevas iniciativas de la política tratan de estimular la cooperación entre los protagonistas públicos, el Estado y regiones, y las empresas. En este nuevo enfoque, se recomienda una estrategia industrial que 
impulse ecosistemas de innovación mediante su desarrollo institucional y que se facilite la coordinación y se incentive la colaboración entre los principales protagonistas de esos sistemas (proveedores, empresas, clientes, centros tecnológicos, instituciones financieras, universidades, centros de formación profesional) para facilitar el aprovechamiento de intangibles colectivos como el conocimiento tecnológico y organizativo y la reputación.

En una línea complementaria, se justifica el apoyo a ecosistemas de emprendimiento (start-ups, capital semilla, capital riego, empresas tractoras que generan spin-offs, mentores, compra pública dinamizadora, universidades, centros de formación) como un marco institucional que favorezca la coordinación y colaboración entre los múltiples protagonistas del emprendimiento.

\section{Conclusiones}

La evidencia mostrada en este trabajo reconoce la persistencia de factores y restricciones en las empresas que dificultan un crecimiento sano y virtuoso de la economía en su conjunto. Las empresas españolas se han construido sobre los viejos paradigmas de gestión y gobierno que se están mostrando crecientemente obsoletos para enfrentarse a los nuevos desafíos de la competencia. Esta debilidad estructural se manifiesta en un dinamismo de la actividad empresarial en España vulnerable, muy sensible a perturbaciones coyunturales y poco resiliente. El decepcionante resultado del crecimiento de la PTF y el estudio y apertura de la caja negra de la empresa (entendida como organización), pone de manifiesto tanto problemas de innovación tecnológica, inversión en activos intangibles, gestión y organización entre las empresas establecidas, como una escasa presencia de nuevas empresas con voluntad de crecer que dinamicen el tejido empresarial. La evolución seguida por la productividad en España no está impulsada por los factores de competitividad de las empresas que han demostrado sus efectos positivos en muchos estudios (buen management, capital organizacional, gestión de las personas, elevadas inversiones en activos intangibles, crecimiento empresarial e intenso esfuerzo innovador). Además, la persistencia de la heterogeneidad en prácticas y resultados dentro del tejido empresarial español, apunta hacia una posible falta de competencia en los mercados de bienes y servicios y de factores, a la que le correspondería expulsar a las empresas poco productivas y dotar de más recursos a las de mayor productividad.

Los desafíos para las empresas españolas son claros. Los retos se vinculan a la capacidad para mejorar su capital emprendedor y directivo que desarrolle las capacidades tecnológicas, humanas y organizativas necesarias para construir un proyecto empresarial ambicioso y competitivo, con voluntad y expectativas de crecimiento.

En este contexto, la política industrial, de la que la política de innovación es una de las más importantes, debe jugar un papel central en la transformación económica que necesitan hacer muchas empresas y, para ello, deberá replantearse sus objetivos e instrumentos de intervención en, al menos, tres aspectos: primero, ampliar el espectro de indicadores de bienestar social desde una visión estrecha de productividad y el crecimiento económico en el pasado a otra más amplia, que incluya también la sostenibilidad medioambiental y la desigualdad en la distribución de la renta y la riqueza. Segundo, reconocer la importancia determinante de las decisiones empresariales en los resultados colectivos, lo que requerirá abandonar la visión reduccionista de la empresa como función de producción, para adoptar otra distinta de la empresa como organización. Tercero, reforzar la función de la política industrial como instrumento que cataliza y coordina el cambio.

\section{Referencias bibliográficas}

Andrés, J. \& Doménech, R. (2015). En busca de la prosperidad: los retos de la sociedad española en la economía global del siglo XXI. Ediciones Deusto.

Autor, D., Dorn, D., Katz, L. F., Patterson, C. \& Van Reenen, J. (2020). The Fall of the Labor Share and the Rise of Superstar Firms. Quarterly Journal of Economics, 135(2), 645-709. 
Bayo Moriones, A. \& Ortín Ángel, P. (2018). Las políticas de recursos humanos en las empresas españolas. Comparación y evolución de las diferencias con otros países de la Unión Europea. En Innovación y competitividad: desafíos para la industria española, capítulo IX (pp. 291-316). FUNCAS.

Bijnens, G. \& Konings, J. (2020). Declining business dynamism in Belgium. Small Business Economics, 54(4), 1201-1239.

Bloom, N. \& Van Reenen, J. (2007). Measuring and Explaining Management Practices across Firms and Countries. The Quarterly Journal of Economics, 122(4), 1351-1408.

Bloom, N. \& Van Reenen, J. (2010). Why do management practices differ across countries? Journal of Economic Perspectives, 24(1), 203-224.

Bloom, N., Lemos, R., Sadun, R., Scur, D. \& Van Reenen, J. (2014). JEEA-FBBVA Lecture 2013: The new empirical economics of management. Journal of the European Economic Association, 12(4), 835-876.

CES, Consejo Económico y Social de España (2020). Cambios tecnológicos, trabajo y actividad empresarial: el impacto socioeconómico de la economía digital.

Corrado, C., Haskel, J., Jona-Lasinio, C. \& lommi, M. (2016). Intangible investment in the EU and US before and since the Great Recession and its contributions to productivity growth. European Investment Bank, Working papers No. 2016/08.

Cuadrado, P., Moral-Benito, E. \& Solera, I. (2020). A Sectoral Anatomy of the Spanish Productivity Puzzle. Banco de España.

De Castro, J. O., Larraza, M. \& Contín, I. (2018). Emprendimiento en España: ilusión vs. realidad. En Innovación y competitividad: desafíos para la industria española, capítulo V (pp. 183-201). FUNCAS.

Decker, R. A., Haltiwanger, J., Jarmin, R. S. \& Miranda, J. (2016). Where has all the Skewness gone? The Decline in High-Growth (Young) Firms in the U.S. European Economic Review, 86(C), 4-23.

ECS, European Company Survey (2019). Eurofound. https://www.eurofound.europa.eu/surveys/2019/ european-company-survey-2019

Foster, L., Haltiwanger, J. \& Krizan, C. J. (2006). Market selection, reallocation, and restructuring in the U.S. retail trade sector in the 1990s. The Review of Economics and Statistics, 88(4), 748-758.

Garcés-Galdeano, L., García-Olaverri, C. \& Huerta, E. (2016). Management capability and performance in Spanish family firms. Academia Revista Latinoamericana de Administración, 29(3), 303-325.

Garcés-Galdeano, L. \& García-Olaverri, C. (2019). The hidden value of intangibles: do CEO characteristics matter? International Journal of Manpower, 40(6), 1075-1091.

Garcés-Galdeano, L. \& Huerta, E. (2019). Las empresas españolas entre el cambio y la inercia: la relevancia de la gestión. España 2018. Un balance. Economistas, 162163, 157-169.

García Perea, P. (2020). Análisis de la Demografía Empresarial en España a Comienzos de 2019. Boletín Económico del Banco de España 2/2020, 1-9.

Giorcelli, M. (2019). The Long-Term effects of management and technology transfers. American Economic Review, 109(1), 121-152.

Haltiwanger, J., Jarmin, R. S., Kulick, R. \& Miranda, J. (2017). High Growth Young Firms: Contribution to Job, Output, and Productivity Growth. National Bureau of Economic Research. University of Chicago Press.

Haskel, J. \& Westlake, S. (2018). Capitalism without Capital: The rise of the Intangible economy. Princeton University Press.

Huerta, E. \& Salas, V. (2014). Tamaño de las Empresas y Productividad de la Economía Española. Un Análisis Exploratorio. Mediterráneo Económico, 25, 167-191.

Huerta, E. \& Salas, V. (2017). Tamaño y Productividad. El Recurso olvidado de la Calidad de dirección. Policy Brief, 12. EuroPe G.

Huerta, E. \& Salas, V. (2018). Productividad y tamaño de las empresas: ¿dónde están las palancas para el cambio? En Innovación y competitividad: desafíos para la industria española, Capítulo XIV (pp. 425-462).

Huerta, E. \& Moral, M. a J. (2018). Innovación y competitividad: desafíos para la industria española. FUNCAS.

IVIE, Instituto Valenciano de Investigaciones Económicas/Fundación BBVA (2014). Crecimiento y Competitividad: Los Desafíos de un Desarrollo Inteligente. Fundación BBVA.

IVIE, Instituto Valenciano de Investigaciones Económicas/Fundación BBVA (2016). Crecimiento y Competitividad: Los Retos de la Recuperación. F. Pérez (dir). Fundación BBVA.

Mas, M. (2020). El crecimiento de la productividad y los activos intangibles. Papeles de Economía Española, 164, 41-59.

Mayer, C. (2020). The Future of the Corporation and the Economics of Purpose. Journal of Management Studies. https://doi.org/10.1111/joms.12660

Mazzucato, M. (2018). Mission-Oriented Research \& Innovation In The European Union. European Commission. DG for Research and Innovation.

Myro, R. (2018). La apuesta por la Industria y la política Industrial. Innovación y competitividad: desafíos para la industria española, capítulo III (pp. 117-139). FUNCAS.

Porter, M. E. (1985). Competitive advantage: Creating and sustaining superior performance. Free Press.

Rubio Benito, N., Villaseñor Román, N. \& Yagüe Guillén, M. J. (2018). El valor de los recursos intangibles para las empresas españolas: el capital directivo. Innovación y competitividad: desafíos para la industria española, capítulo IV (pp. 143-181). FUNCAS. 
Schivardi, F. \& Schmitz, T. (2019). The IT Revolution and Southern Europe's Two Lost Decades. Journal of the European Economic Association, 18(5), 2441-2486.

Schumpeter, J. (1939). Business cycles. McGraw-Hill.

Stiglitz, J. E. (1994). Economics growth revisited. Industrial and Corporate Change, 3(1), 65-110.
Syverson, C. (2011). What Determines Productivity? Journal of Economic Literature, 49(2), 326-365.

Warwick, K. (2013). Beyond Industrial Policy: Emerging Issues and New Trends. OECD Science, Technology and Industry Policy Papers, No. 2. OECD Publishing Paris. 